\title{
Real-world data on the survival outcome of patients with newly diagnosed Waldenström macroglobulinemia
}

Jang Ho Cho ${ }^{1,2, *}$, Joon-Ho Shim ${ }^{3,4,}$, Sang Eun Yoon ${ }^{2}$, Hee-Jin $\mathrm{Kim}^{5}$, Sun-Hee Kim ${ }^{5}$, Young Hyeh $\mathrm{Ko}^{6}$, Seung-Tae Lee ${ }^{7}$, Kihyun $\mathrm{Kim}^{2}$, Won Seog Kim ${ }^{2}$, and Seok Jin Kim ${ }^{2,4}$

\begin{abstract}
${ }^{1}$ Division of Oncology, Department of Internal Medicine, Incheon St. Mary's Hospital, College of Medicine, The Catholic University of Korea, Incheon; ${ }^{2}$ Division of Hematology-Oncology, Department of Medicine, ${ }^{3}$ Samsung Genome Institute, Samsung Medical Center, Sungkyunkwan University School of Medicine, Seoul; ${ }^{4}$ Department of Health Science and Technology, Samsung Advanced Institute of Health Science and Technology, Sungkyunkwan University, Seoul; Departments of ${ }^{5}$ Laboratory Medicine \& Genetics and ${ }^{6}$ Pathology, Samsung Medical Center, Sungkyunkwan University School of Medicine, Seoul; ${ }^{7}$ Department of Laboratory Medicine, Yonsei University College of Medicine, Seoul, Korea
\end{abstract}

Received: November 2, 2019

Revised : January 13, 2020

Accepted: January 31, 2020

\section{Correspondence to}

Seok Jin Kim, M.D.

Division of Hematology-

Oncology, Department of

Medicine, Samsung Medical

Center, Sungkyunkwan

University School of Medicine,

81 Irwon-ro, Gangnam-gu, Seoul

06351, Korea

Tel: +82-2-3410-1766

Fax: +82-2-3410-1754

E-mail: kstwoh@skku.edu

https://orcid.org/0000-0002-

$2776-4401$

*These authors contributed equally to this work.

Background/Aims: Waldenström macroglobulinemia (WM) is a rare lymphoproliferative disorder that usually follows an indolent clinical course. However, some patients show an aggressive clinical course leading to death. We explored the risk factors predicting poor prognosis in WM patients.

Methods: We retrospectively analyzed 47 patients diagnosed with WM between 2000 and 2018 to explore risk factors predicting poor prognosis using various clinical and laboratory parameters and risk models including the International Prognostic Staging System for WM (IPSS-WM).

Results: Over a median follow-up duration of 80.4 months, 29 patients died. The main causes of death were disease progression, organ failure related to amyloidosis, and infection. The median overall survival (OS) was 55.1 months, and 14 patients, including three with amyloidosis, died within 2 years. Serum $\beta 2$ microglobulin level higher than $4 \mathrm{mg} / \mathrm{dL}$ was significantly associated with poor OS. Accordingly, the IPSS-WM showed a significant association with poor prognosis compared with other risk models, and the low-risk group had better OS than intermediate- and high-risk groups. In the retrospective analysis using the results of targeted sequencing in two cases representing good and bad prognosis, different patterns of mutation profiles were observed, including mutations of MYDS8, TP53, ARID1A, and JAK2 in a refractory case.

Conclusions: Serum $\beta 2$-microglobulin could be a single biomarker strongly predictive of poor survival of WM patients, and the low-risk group of the IPSS-WM risk model including serum $\beta 2$-microglobulin has better prognostic value than other risk models. Mutation analysis also might provide additional information to predict high-risk patients.

Keywords: Waldenstrom macroglobulinemia; Amyloidosis; Survival; Rituximab

\section{INTRODUCTION}

Waldenström macroglobulinemia(WM) is a rare lymphoproliferative disorder, with a worldwide incidence of three to five cases per million persons per year [1]. The diagnosis of WM requires immunoglobulin $\mathrm{M}$ ( $\operatorname{IgM})$ monoclonal gammopathy of any concentration and bone marrow infiltration by lymphoplasmacytic 
lymphoma (LPL) cells [2]. WM generally occurs in elderly people and follows an indolent clinical course with median survival of 50 to 60 months [3]. Thus, many patients with WM may be asymptomatic at diagnosis, and urgent therapy might not be required in most cases. The Second International Workshop on WM recommended initiating therapy in WM patients with constitutional symptoms, symptomatic lymphadenopathy or splenomegaly, anemia (hemoglobin $\leq 10 \mathrm{~g} / \mathrm{dL}$ ) or thrombocytopenia (platelet count $<100 \times 109 / \mathrm{L}$ ), and complications related to increased level of IgM such as neuropathy and amyloid light chain (AL) amyloidosis [4].

Various drugs have been used as primary treatment for newly diagnosed WM, from classical alkylating agents to monoclonal antibodies such as rituximab, and a recent meta-analysis showed that rituximab-based immunochemotherapy could be highly effective for WM, with tolerable toxicities [5]. Furthermore, the use of novel targeted agents such as the Bruton tyrosine-kinase inhibitor ibrutinib improves the outcome of WM patients, according to a recent phase III study comparing ibrutinib and rituximab with rituximab alone [6]. However, early disease progression and death may occur in some patients even though the recent Swedish Lymphoma Registry between 2000 and 2014 showed median overall survival of 96 months [7]. Although they might account for a small portion of WM patients, the identification of patients at high risk of early progression and death is important to prevent treatment failure.

For prognostication in WM patients, the International Prognostic Staging System for WM (IPSS-WM) based on disease parameters evaluated at the time of first-line treatment is the most widely accepted prognostic index, consisting of age $>65$ years, hemoglobin $\leq 11.5 \mathrm{~g} /$ $\mathrm{dL}$, platelet count $\leq 100 \times 10^{9} / \mathrm{L}$, serum $\beta 2$-microglobulin $\geq 3 \mathrm{mg} / \mathrm{dL}$, and serum monoclonal protein $>7 \mathrm{~g} / \mathrm{dL}$ [8]. Other prognostic models have been proposed consisting of parameters similar to that of IPSS-WM, such as age or hemoglobin and $\beta 2$-microglobulin levels [9-11]. Furthermore, a recent study proposed a progression risk classification of asymptomatic WM (AWM risk) patients using bone marrow LPL cells greater than 70\%, increased IgM level higher than $4.5 \mathrm{~g} / \mathrm{dL}$, albumin less than $3.5 \mathrm{~g} / \mathrm{dL}$, and serum $\beta 2$-microglobulin $\geq 4 \mathrm{mg} / \mathrm{dL}$ [12]. Thus, we analyzed the feasibility of those risk models to identify patients at high risk of progression and death and explored parameters predicting poor prognosis in WM patients.

\section{METHODS}

We reviewed the electronic medical records of patients who were pathologically diagnosed with lymphoma and plasma cell neoplasm at Samsung Medical Center between 2000 and 2018 and searched for the term 'lymphoplasmacytic lymphoma' or 'Waldenström macroglobulinemia.' Among 72 patients diagnosed with LPL or WM, we identified 55 patients with WM after excluding LPL patients without IgM monoclonal gammopathy. As the purpose of this study was to explore parameters predicting poor prognosis based on baseline clinical and laboratory characteristics in WM patients, we selected only symptomatic WM patients requiring treatment. Thus, we excluded eight patients with asymptomatic WM who did not receive treatment after initial diagnosis. Ultimately, we analyzed 47 symptomatic WM patients and collected parameters at diagnosis known to be related to prognosis, including age at diagnosis, Eastern Cooperative Oncology Group (ECOG) performance status, percentage of bone marrow LPL cells, hemoglobin level, platelet count, serum albumin, $\beta 2$-microglobulin, and serum lactate dehydrogenase levels. We further obtained information on presenting clinical manifestations including constitutional symptoms, lymphadenopathy, and hepatosplenomegaly, as well as the site of involvement and presence of AL amyloidosis. Clinical and laboratory characteristics were analyzed, and the best response to the first-line treatment was compared according to the response criteria recommended from the Third International Workshop on WM [13].

We also gathered the results of targeted sequencing from the data registry of our prospective cohort after written informed consent (ClinicalTrials.gov Identifier: NCTo1877109). Targeted sequencing was performed with paraffin-embedded tissue samples using the $\mathrm{He}$ maSCAN containing 425 genes related to hematological malignancies (Supplementary Table 1) [14]. Thus, we retrospectively analyzed the mutation profiles of two representative cases to compare the distribution of mutations between good and poor prognoses. Detailed methods have been described previously [14,15]. Briefly, genomic DNA was extracted using a QIAamp DNA Mini 

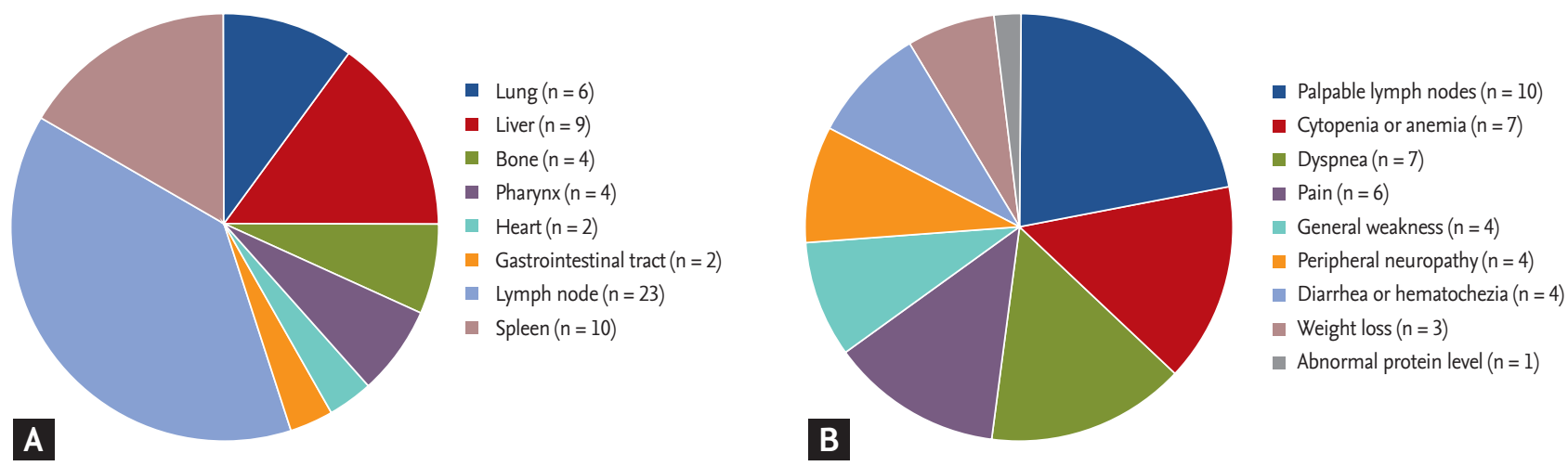

Figure 1. (A) Distribution of involved sites. (B) Frequency of clinical presentation.

kit (Qiagen, Valencia, CA, USA). The mean sequencing coverage was greater than 70ox. Somatic alterations including mutations, copy number alteration, and structure variants were called using a previously described pipeline: MuTect version 1.1.6, Lowfreq version 0.6.1, Pindel version 0.2.5a4 software, and a custom-built inhouse algorithm were used [15-17].

For statistical analysis, the chi-square test was used for comparison of characteristics, the Kaplan-Meier method was used for univariate analysis of survival outcomes, and the log-rank test was used for comparisons. Cox regression hazard analysis was also performed for multivariate analysis of overall survival. Overall survival was measured from the date of diagnosis to the date of death from any cause and was censored at the date of the last follow-up visit. Statistical associations were determined by the log-rank test. Two-sided $p$ values $<0.05$ were considered significant. All analyses were performed using SPSS version 23.0 (IBM SPSS Inc., Armonk, NY, USA) and R3.6.1 software. This study was approved by the Institutional Review Board of Samsung Medical Center, Seoul, Korea, and the requirement for informed consent was waived because of the retrospective nature of the study (No. 2018-06-149). All procedures performed in studies involving human participants were in accordance with the ethical standards of the institutional and/or national research committee and with the 1964 Helsinki declaration and its later amendments or comparable ethical standards.

\section{RESULTS}

\section{Characteristics of patients at diagnosis}

The median age of the 47 patients was 68 years (range, 27 to 86) at diagnosis, and patients over 65 years old accounted for $62 \%$ (29/47) of patients. As all patients were referred from primary physicians or a secondary hospital to our center, a tertiary hospital, most patients had symptoms and/or signs associated with WM, including symptomatic lymphadenopathy, dyspnea, cytopenia, neuropathy, and constitutional symptoms such as weakness. However, the frequency of B symptoms was very low $(6 \%, 3 / 47)$. Accordingly, most patients showed good performance status (ECOG PS o/1, 85\%, 40/47). Lymphadenopathy was observed in half of the patients $(49 \%$, 23/47), and 16 patients (34\%) showed $\geq 2$ involved extranodal sites. Hepatomegaly and/or splenomegaly were found in $38 \%$ of patients (18/47), and one other involved extranodal sites included the lung and gastrointestinal tract (Fig. 1A). Clinical manifestations at the time of initial visit to the clinic were variable and included lymph node enlargement, dyspnea, weakness, and peripheral neuropathy (Fig. 1B). The median percentage of tumor cells in bone marrow aspirates was $35 \%$ (range, 10\% to $90 \%)$. More than half of the patients $(57 \%, 27 / 47)$ had a hemoglobin level lower than $10 \mathrm{~g} / \mathrm{dL}$, while only nine patients had thrombocytopenia (platelet count $<100 \times$ 109/L, 19\%, 9/47). The presence of cold agglutinin was not initially evaluated in most patients, and level of hemoglobin and thrombocytopenia was not significantly associated with percentage of tumor cells in the bone 

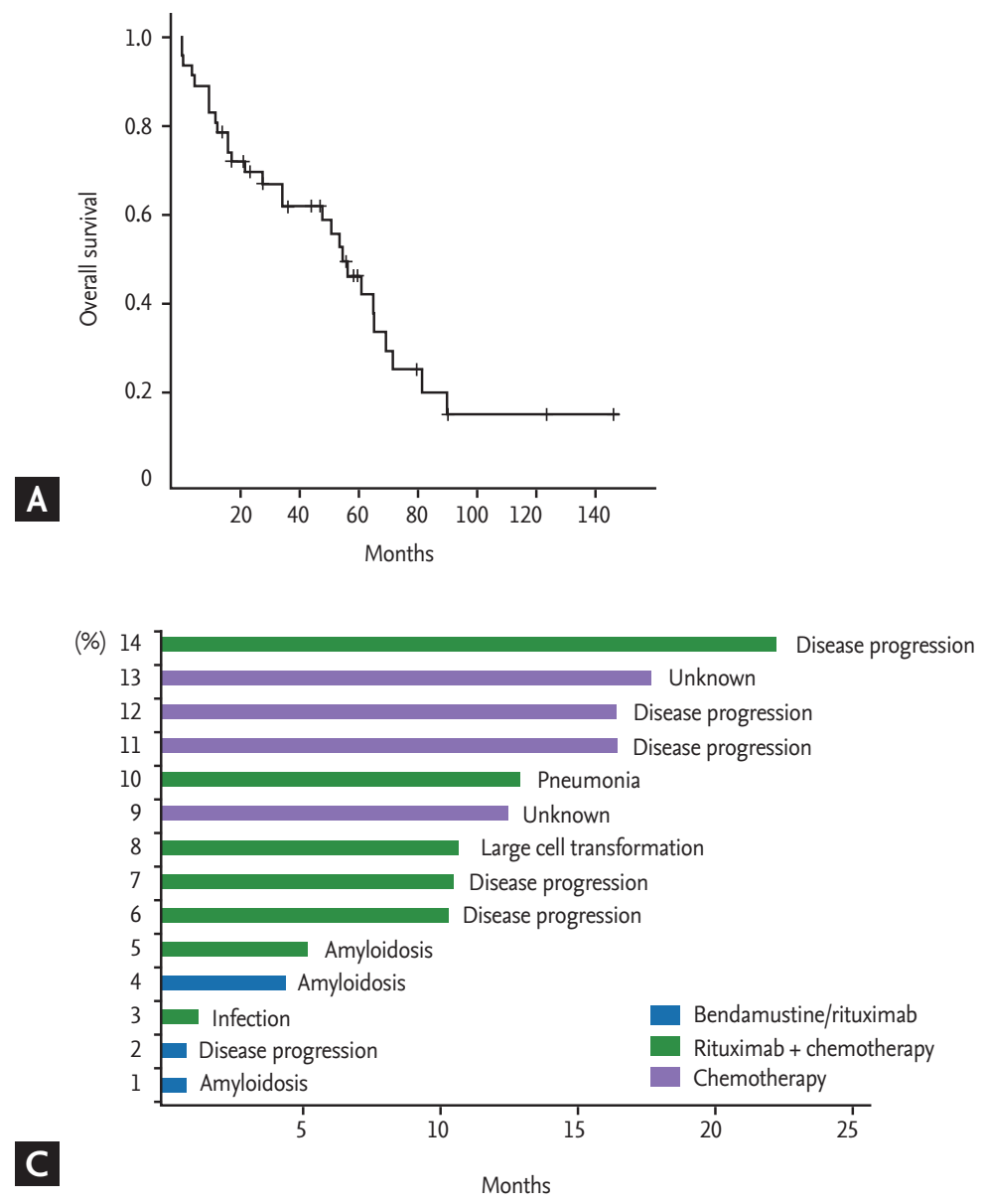
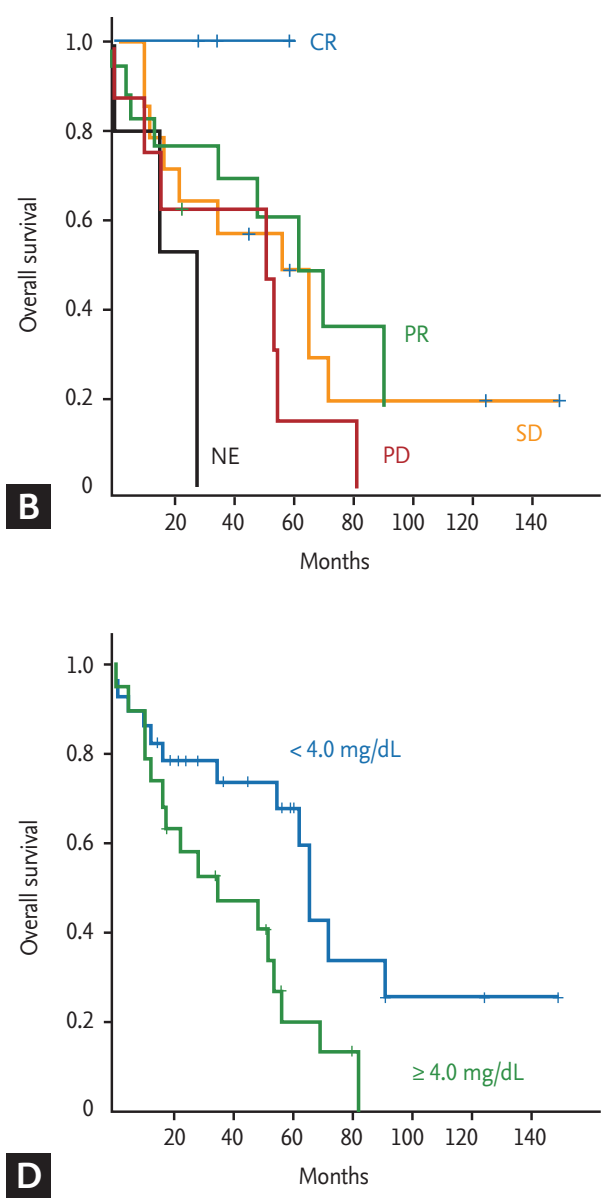

Figure 2. (A) Overall survival. (B) Comparison of overall survival by response to initial treatment. (C) Survival duration and cause of death in patients who died within 2 years of diagnosis. (D) Survival differences according to high and low serum $\beta 2-$ microglobulin level. CR, complete response; PR, partial response; SD, stable disease; PD, progressive disease; NE, not evaluated.

marrow (data not shown). All patients had IgM monoclonal gammopathy, and the level of IgM was variable (median, 3,614 mg/dL; range, 316 to 10,795). However, there were no cases with hyperviscosity symptoms such as headache. Decreased serum albumin (median, $3.6 \mathrm{~g} /$ $\mathrm{dL}$; range, 2.3 to 4.4 ) and increased $\beta 2$-microglobulin levels (median, $3.6 \mathrm{mg} / \mathrm{dL}$; range, 1.1 to 9.1) were also observed. Three patients with AL amyloidosis presenting with neuropathic pain or diarrhea were finally diagnosed with WM after bone marrow aspiration and immune phenotyping analysis. However, not all patients received a systemic evaluation sufficient to exclude the presence of AL amyloidosis including biopsy at the time of diagnosis based on the review of medical records. Thus, the exact frequency of AL amyloidosis in WM patients could not be determined by the data of this study.

\section{Treatment and survival outcomes}

Because use of rituximab was not approved by the Korean Health Insurance System before 2013, only 19 patients received rituximab-containing immunochemotherapy according to previously reported protocols [18,19]: R-CHOP (rituximab, cyclophosphamide, doxorubicin, vincristine, and prednisone, $\mathrm{n}=6$ ), R-CVP (rituximab, cyclophosphamide, vincristine, and prednisone, $\mathrm{n}=1$ ), R-CD (rituximab, cyclophosphamide, and dexamethasone, $\mathrm{n}=2$ ), or BR (bendamustine plus rituximab, $\mathrm{n}=$ 10). The remaining 28 patients who were diagnosed with WM before 2013 received alkylator chemotherapy such as chlorambucil ( $n=16$ ) or cyclophosphamide plus prednisolone $(n=12)$. Out of 10 patients receiving $B R$, nine responded (complete response [CR] 2, partial response $[\mathrm{PR}] 7$ ), whereas five patients responded to other ritux- 
Table 1. Clinical features and outcome of patients with presence of amyloid

\begin{tabular}{|c|c|c|c|}
\hline Variable & Male/76 yr & Female/76 yr & Male/5o yr \\
\hline Immunophenotype & IgM/kappa & IgM/lambda & IgM/kappa, lambda \\
\hline Initial symptom & Diarrhea & Chest pain & Diarrhea \\
\hline Paraproteinemia, mg/dL & $\operatorname{IgM}_{1}, 831$ & $\operatorname{IgM} 2,348$ & $\operatorname{IgM}_{5,630}$ \\
\hline Serum monoclonal protein, g/dL & 0.19 & 1.2 & $3 \cdot 3$ \\
\hline Bone marrow involvement, \% & 60 & 70 & 70 \\
\hline Involved organ & $\begin{array}{l}\text { Gastrointestinal tract, } \\
\text { heart, nerve }\end{array}$ & Heart, nerve & $\begin{array}{l}\text { Gastrointestinal tract, } \\
\text { heart, liver, nerve }\end{array}$ \\
\hline NT-proBNP, pg/mL & 355.8 & 6,001 & 906.4 \\
\hline Troponin T, ng/mL & 0.0027 & 0.084 & 0.014 \\
\hline First-line treatment & Bendamustine, rituximab & $\begin{array}{l}\text { Bendamustine, } \\
\text { rituximab }\end{array}$ & $\begin{array}{l}\text { Rituximab, cyclophosphamide, } \\
\text { dexamethasone }\end{array}$ \\
\hline Hematologic response & Partial response & Partial response & Partial response \\
\hline Organ response & None & None & None \\
\hline Overall survival, mon & 0.8 & $4 \cdot 5$ & $5 \cdot 3$ \\
\hline Survival & Dead & Dead & Dead \\
\hline Cause of death & Sepsis and heart failure & Heart failure & Sepsis \\
\hline
\end{tabular}

IgM, immunoglobulin M; NT-proBNP, natriuretic peptide pro-brain natriuretic peptide.

imab-containing immunochemotherapy ( $\mathrm{CR} 1$ 1, PR 4). Among the 19 patients receiving rituximab-containing immunochemotherapy, there was no case showing laboratory findings suspicious of IgM flare. The response of alkylator chemotherapy was not satisfactory; only six patients showed $\mathrm{PR}$, while the remaining patients showed stable disease (SD; $n=16)$ or progression $(n=6)$. With a median follow-up duration of 80.4 months (95\% confidence interval [CI], 45.8 to 115.0), 29 patients died due to disease progression $(n=18)$, organ failure related to amyloidosis $(n=3)$, infection $(n=4)$, unknown cause $(\mathrm{n}=3)$, and lung cancer $(\mathrm{n}=1)$. The median OS was 55.1 months at the time of analysis (95\% CI, 43.3 to 66.8) (Fig. $2 A)$. Although the number of patients in each treatment group was too small for statistical significance, complete responders to the first-line treatment showed better overall survival (OS) than patients with PR and other responses (Fig. 2B). Among the 47 patients, 14 died within 2 years of the first diagnosis. Their cause of death was mainly associated with disease, including presence of amyloidosis regardless of first-line treatment regimen (Fig. 2C). Patients with amyloidosis who failed to show organ response and clinical symptoms such as pain, diarrhea, and heart failure did not improve, even though serum level of immunoglobulin decreased after chemotherapy. They eventually died due to organ failure related to amyloidosis (Table 1).

\section{Risk factor analysis}

Clinical and laboratory characteristics at diagnosis were compared according to the final survival outcome (Table 2). Increased serum $\beta 2$-microglobulin level (> $4 \mathrm{mg} /$ $\mathrm{dL}$ ) wase more frequently found in patients who died compared to surviving patients $(p=0.014)$. However, there were no other parameters significantly associated with occurrence of death even though we performed statistical analyses using various cutoff values for IgM level, hemoglobin, albumin, and percentage of bone marrow LPL cells. The cutoff of IgM ( $4.5 \mathrm{~g} / \mathrm{dL}$ ) and bone marrow LPL cells (70\%) in the progression risk classification of asymptomatic WM was also not related to the occurrence of death (Table 2). Accordingly, serum 32-microglobulin level higher than $4 \mathrm{mg} / \mathrm{dL}$ was significantly associated with OS $(p=0.015)$ (Fig. $2 \mathrm{D})$. Among four risk models applied to our patients, IPSS-WM risk and Mayo risk models showed high incidence of death in patients designated as high-risk. However, as $75 \%$ of patients belonged to the high-risk group of the Mayo 
Table 2. Characteristics of patients at diagnosis

\begin{tabular}{|c|c|c|c|c|}
\hline Characteristic & All patients $(n=47)$ & Alive $(n=18)$ & Death $(\mathrm{n}=29)$ & $p$ value \\
\hline Sex & & & & 0.108 \\
\hline Male & $33(70)$ & $10(30)$ & $23(70)$ & \\
\hline Female & $14(30)$ & $8(57)$ & $6(43)$ & \\
\hline Age, yr & & & & 0.229 \\
\hline$\leq 65$ & $18(38)$ & $9(50)$ & $9(50)$ & \\
\hline$>65$ & $29(62)$ & $9(31)$ & $20(69)$ & \\
\hline ECOG PS & & & & 0.692 \\
\hline o/1 & $40(85)$ & $16(40)$ & $24(60)$ & \\
\hline$\geq 2$ & $7(15)$ & $2(29)$ & $5(71)$ & \\
\hline Serum LDH & & & & 0.219 \\
\hline Normal & $36(77)$ & $16(44)$ & $20(56)$ & \\
\hline Increased & $8(17)$ & $2(25)$ & $6(75)$ & \\
\hline Unknown & $3(6)$ & o & $3(100)$ & \\
\hline Lymphadenopathy & & & & 0.556 \\
\hline Absence & $24(51)$ & $8(33)$ & $16(67)$ & \\
\hline Presence & $23(49)$ & $10(44)$ & $13(56)$ & \\
\hline Hepatosplenomegaly & & & & 0.356 \\
\hline Absence & $29(62)$ & $13(45)$ & $16(55)$ & \\
\hline Presence & $18(38)$ & $5(28)$ & $13(72)$ & \\
\hline Albumin & & & & 0.122 \\
\hline$\geq 3.5 \mathrm{~g} / \mathrm{dL}$ & $29(62)$ & $14(48)$ & $15(52)$ & \\
\hline$<3.5 \mathrm{~g} / \mathrm{dL}$ & $18(38)$ & $4(22)$ & $14(78)$ & \\
\hline$\beta 2$-microglobulin & & & & 0.014 \\
\hline$\leq 4 \mathrm{mg} / \mathrm{dL}$ & $28(60)$ & $15(54)$ & $13(46)$ & \\
\hline$>4 \mathrm{mg} / \mathrm{dL}$ & $19(40)$ & $3(16)$ & $16(84)$ & \\
\hline Hemoglobin & & & & 0.226 \\
\hline$>10 \mathrm{~g} / \mathrm{dL}$ & $20(43)$ & $10(50)$ & $10(50)$ & \\
\hline$\leq 10 \mathrm{~g} / \mathrm{dL}$ & $27(57)$ & $8(30)$ & $19(70)$ & \\
\hline Platelet & & & & 0.449 \\
\hline$>100,000 / \mathrm{L}$ & $38(81)$ & $16(42)$ & $22(58)$ & \\
\hline$\leq 100,000 / \mathrm{L}$ & $9(19)$ & $2(22)$ & $7(78)$ & \\
\hline Albumin & & & & 0.726 \\
\hline$\geq 4 \mathrm{~g} / \mathrm{dL}$ & $11(23)$ & $5(46)$ & $6(54)$ & \\
\hline$<4 \mathrm{~g} / \mathrm{dL}$ & $36(77)$ & $13(36)$ & $23(64)$ & \\
\hline IgM & & & & 0.111 \\
\hline$<4.5 \mathrm{~g} / \mathrm{dL}$ & $32(68)$ & $15(47)$ & $17(53)$ & \\
\hline$\geq 4.5 \mathrm{~g} / \mathrm{dL}$ & $15(32)$ & $3(20)$ & $12(80)$ & \\
\hline B symptoms & & & & 0.276 \\
\hline Absence & $44(94)$ & $18(41)$ & $26(59)$ & \\
\hline Presence & $3(6)$ & o & $3(100)$ & \\
\hline Amyloidosis & & & & 0.276 \\
\hline Unknown & $44(94)$ & $18(41)$ & $26(59)$ & \\
\hline
\end{tabular}


Table 2. Continued

\begin{tabular}{|c|c|c|c|c|}
\hline Characteristic & All patients $(n=47)$ & Alive $(n=18)$ & Death $(n=29)$ & $p$ value \\
\hline Presence & $3(6)$ & 0 & $3(100)$ & \\
\hline Bone marrow tumor cell & & & & 0.716 \\
\hline$<70 \%$ & $38(81)$ & $14(37)$ & $24(63)$ & \\
\hline$\geq 70 \%$ & $9(19)$ & $4(44)$ & $5(56)$ & \\
\hline IPSS-WM risk & & & & 0.003 \\
\hline Low & $4(8)$ & $4(100)$ & o & \\
\hline Intermediate & $23(49)$ & $11(48)$ & $12(52)$ & \\
\hline High & $20(43)$ & $3(15)$ & $17(85)$ & \\
\hline French group risk & & & & 0.186 \\
\hline Low & $6(13)$ & $4(67)$ & $2(33)$ & \\
\hline Intermediate & $16(34)$ & $7(44)$ & $9(56)$ & \\
\hline High & $25(53)$ & $7(28)$ & $18(72)$ & \\
\hline Mayo risk & & & & 0.024 \\
\hline Low & $4(16)$ & $4(100)$ & 0 & \\
\hline Intermediate & $5(9)$ & $1(20)$ & $4(80)$ & \\
\hline High & $38(75)$ & $13(34)$ & $25(66)$ & \\
\hline SWOG risk & & & & 0.138 \\
\hline Low & $7(15)$ & $5(71)$ & $2(29)$ & \\
\hline Medium & $26(55)$ & $9(35)$ & $17(65)$ & \\
\hline High & $14(30)$ & $4(29)$ & $10(71)$ & \\
\hline Treatment & & & & 0.065 \\
\hline BR & $10(21)$ & $7(70)$ & $3(30)$ & \\
\hline R-CTx & $9(19)$ & $3(33)$ & $6(67)$ & \\
\hline CTx & $28(60)$ & $8(28)$ & $20(71)$ & \\
\hline
\end{tabular}

Values are presented as number (\%).

ECOG, Eastern Cooperative Oncology Group; PS, performance status; LDH, lactate dehydrogenase; IgM, immunoglobulin M; IPSS-WM, International Prognostic Staging System for Waldenström macroglobulinemia; SWOG, Southwest Oncology Group; BR, bendamustine, rituximab; R-CTx, rituximab-chemotherapy; CTx, chemotherapy.

risk model, its clinical relevance was lower than that of the IPSS-WM risk model, designating $43 \%$ of patients as high-risk (Table 2). However, the comparison of OS based on risk model showed that only low-risk patients had better OS than intermediate- and high-risk patients $(p<0.05)$, whereas there was no difference between intermediate- and high-risk patients in the IPSS-WM risk model (Fig. 3A). The association of other risk models with OS was not significant (Fig. 3B-3D).

\section{Mutation analysis}

Although evaluation of the MYDS8 L265P mutation was not performed in all patients at diagnosis, our previous analysis using the mutant enrichment 3'-modified oligonucleotide-polymerase chain reaction technique found 21 out of 28 LPL cases (75\%) with the MYD88 L265P mutation in bone marrow aspirates [20]. In addition to the above-mentioned 21 cases, we performed targeted sequencing using paraffin-embedded tissue blocks from two representative cases from our study population. One case (male/68 years old with IgM/kappa) showed early death within 2 years after diagnosis. Although he was treated with rituximab-CHOP chemotherapy immediately after diagnosis, disease progression occurred after the fifth cycle and became refractory to subsequent salvage chemotherapies. The other case (female/69 years 


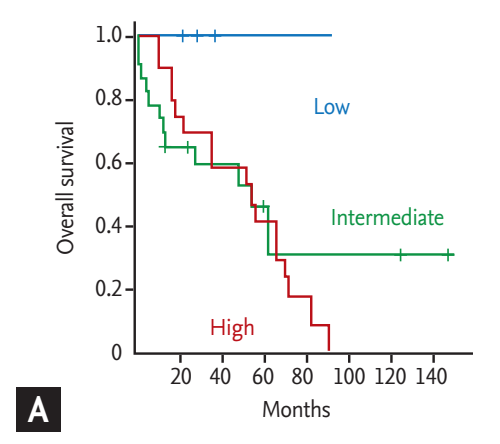

\section{IPSS WM}

Low: 0 or 1 factor (except age)

Intermediate: age or 2 factors

High: $\geq 3$ factors

Age $>65$ years

Hemoglobin $\leq 11.5 \mathrm{~g} / \mathrm{dL}$

Platelet $\leq 100 \times 10^{9} / \mathrm{L}$

$\beta 2$ microglobulin $>3 \mathrm{mg} / \mathrm{dL}$

Serum monoclonal protein $>7 \mathrm{~g} / \mathrm{dL}$
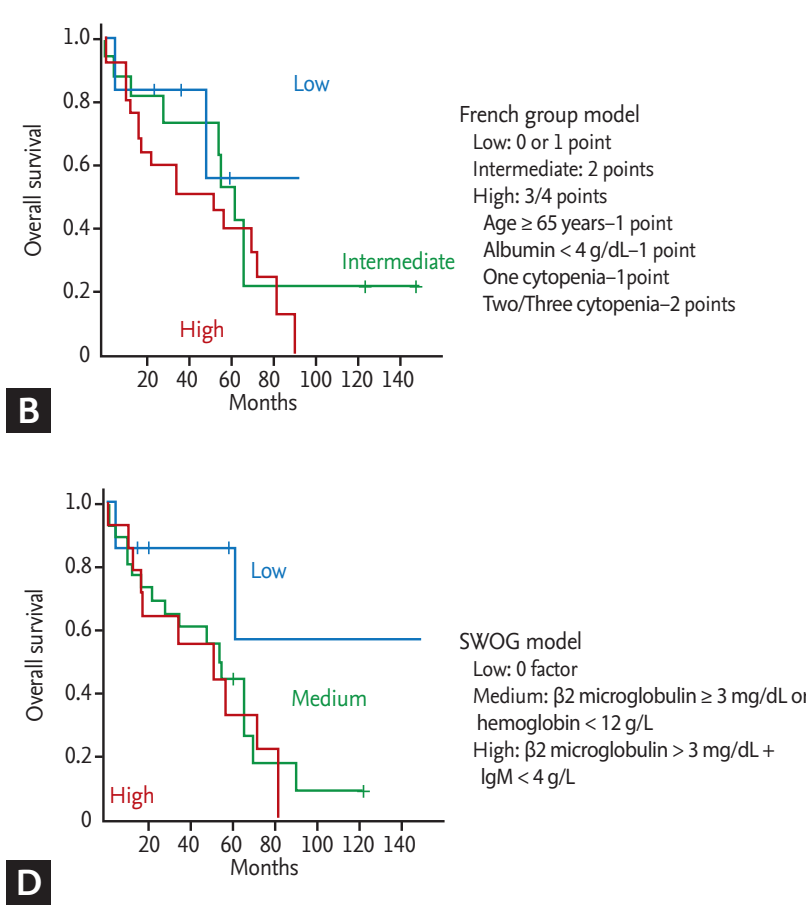

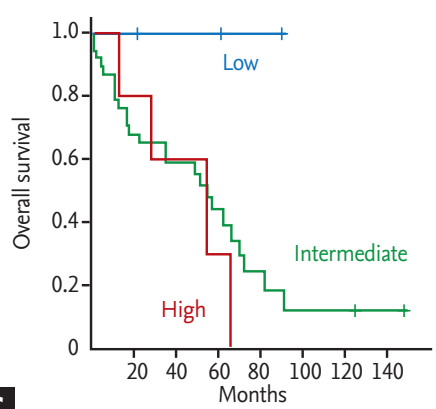

Mayo model

Low: 0 factor

Intermediate: 1 factor

High: $\geq 2$ factors

Age $>65$ years

Presence of organomegaly

$\beta 2$ microglobulin $\geq 4 \mathrm{mg} / \mathrm{dL}$

Figure 3. (A) Survival comparison by the International Prognostic Staging System for Waldenström macroglobulinemia (IPSS-WM), (B) French group model, (C) Mayo model, and (D) Southwest Oncology Group (SWOG) model. IgM, immunoglobulin M.

old with IgM/lambda) survived. Although she experienced relapse 3 years after completion of her first-line treatment (R-CHOP), she responded to the salvage chemotherapy including rituximab. Comparison of sequences revealed differences between the two cases, including mutations of MYDS8, TP53, ARID1A, and JAK2 in the ED case (Fig. 4A).

\section{DISCUSSION}

WM is an extremely rare disease in Asian countries, and most data are from Western patients. Indeed, a nationwide analysis of the incidence of malignant lymphoma according to the WHO classification between 2005 and 2006 reported an incidence rate of $0.3 \%$ in Korea [21]. The clinical course of WM is variable, ranging from asymptomatic cases with increased IgM to symptomatic cases with cytopenia and organomegaly. Thus, approximately $40 \%$ of WM patients have a mild form of anemia; other non-specific symptoms may include weakness, fatigue, and weight loss. One-third of patients may have lymph node enlargement and hepatosplenomegaly. As a substantial number of patients with WM follow an indolent course without progression to an aggressive state for a long time, treatment initiation should not be based on serum IgM level. Instead, a 'watch and wait' strategy could be considered until patients develop symptoms requiring therapy. However, 29 patients had died at the time of analysis in our study, and the majority was due to disease progression. As 14 patients died within 2 years of diagnosis, the median OS was 55.1 months, which was lower than that of a recently published Swedish nation-wide dataset reporting a median OS of 96 months [7]. This difference might be associated with the symptomatic aggressive WM in the majority of patients in our study, as mentioned above. Furthermore, most patients who were diagnosed with WM before 2013 received chemotherapy with alkylators due to reimbursement issues with rituximab-containing immunochemotherapy. Indeed, their response was poor (PR 6, SD 16, and progressive disease 6), which may have led to inferior outcomes for our patients. Currently, alkylators or nucleoside analogues are not recommended for patients younger than 65 years to avoid secondary malignancies and disease transformation, and rituximab-containing immu- 


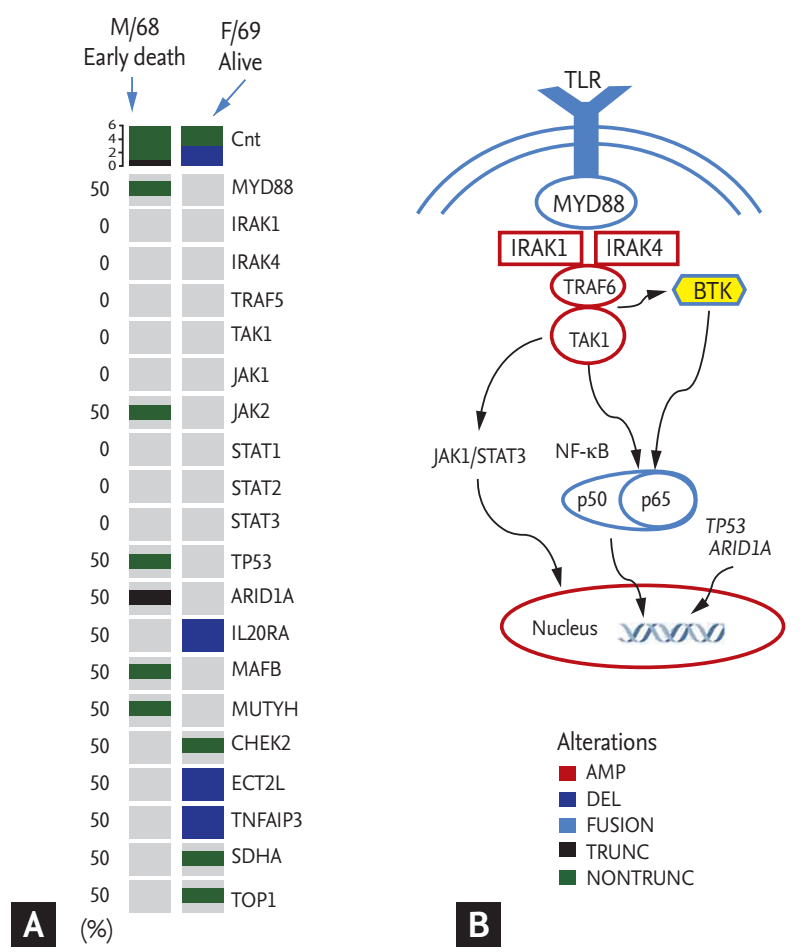

Figure 4. (A) Heatmap illustrating genetic alterations detected in the two representative cases. Mutations in MYDSS, TP53, ARID1A, and JAK2 were identified in the early death case (male/68 yr). (B) Signaling pathway related to MYDS8, TP53, and ARID1A. TLR, Toll-like receptor; MYD88, myeloid differentiation primary response 88; IRAK, interleukin-1 receptor (IL-1R) associated kinase; TRAF6, tumor necrosis factor receptor associated factor 6 ; TAK1, transforming growth factor- $\beta$-activated kinase 1 ; JAK1, Janus kinase 1 ; STAT3, signal transducer and activator of transcription 3 ; NF- $\mathrm{KB}$, nuclear factor-kappa B; BTK, Bruton tyrosine kinase; $\mathrm{TP}_{53}$, tumor protein 53; ARIDIA, AT-rich interactive domain-containing protein $1 \mathrm{~A}$; AMP, amplification; DEL, deletion; TRUNC, truncated mutation; NONTRUNC, non-truncated mutation.

nochemotherapy regimens have become the mainstay of treatment [22]. In particular, a phase III non-inferiority study comparing BR with R-CHOP as the first-line treatment reported that $\mathrm{BR}$ is associated with longer progression-free survival (69 months vs. 29 months) and better tolerance in WM patients [19]. Although we could not show significant difference of OS according to the type of treatment due to the small number of patients in each treatment group and the retrospective nature of our study, the BR regimen could be one treatment option for WM patients like the currently preferred R-CD regimen, considering its efficacy and tolerable toxicity compared to R-CHOP [23].

In this study, we evaluated the predictive value of IPSS-WM and other prognostic models for predicting the poor prognosis in WM patients [8-11]. However, the comparison of OS based on risk model showed that only low-risk patients had better OS than intermediateand high-risk patients, whereas there was no difference between intermediate- and high-risk patients in the IPSS-WM risk model (Fig. 3A). In addition, prognostic values of other risk models were less than we expected in Korean WM patients. When we performed univariate analysis using previously reported prognostic factors, including age more than 65 years, presence of cytopenia, serum IgM level, percentage of bone marrow tumor cells, and poor performance status, only serum $\beta 2$-microglobulin level higher than $4 \mathrm{mg} / \mathrm{dL}$ was significantly associated with OS. Given that serum 32-microglobulin level is included as a component of three prognostic models (IPSS-WM, Mayo, and Southwest Oncology Group [SWOG]), measurement of serum ß2-microglobulin might be useful for predicting poor survival outcome of WM as a single biomarker. However, our study has several limitations. First, treatment regimens were heterogeneous, and the number of patients in each treatment was too small to draw a solid conclusion. Second, our results could be influenced by selection bias due to the retrospective nature of this single-institute study. Accordingly, multivariate analysis could not be performed. Further studies with a larger study population should be performed to evaluate the prognostic value of other parameters such as serum IgM level and bone marrow tumor cells considering their potential association with poor prognosis of WM. The increased level of IgM could induce amyloid deposits, resulting in light chain (AL) amyloidosis, and IgM-related amyloidosis is present in $5 \%$ to $7 \%$ of patients with AL amyloidosis $[24,25]$. In this study, three patients with AL amyloidosis died due to organ failure related to amyloidosis, even though they all showed a hematologic response to BR or R-CD (Table 1). However, systemic evaluation for the presence of AL amyloidosis was not performed in most patients of this study because amyloidosis is a relatively uncommon event. Thus, the prognostic value of AL amyloidosis in WM patients also should be confirmed in a further prospective study.

As data regarding genomic alterations of WM accu- 
mulate, genomics-based prognostication has been tried. Although the MYDS8 L265P mutation can be found to a lesser extent in other indolent or aggressive lymphomas such as marginal zone lymphoma and diffuse large B-cell lymphoma, whole-genome sequencing of bone marrow tumor cells reveals MYDS8 L265P as a frequent mutation in patients with WM [26]. Better OS has been reported in patients with the MYDS8 L265P mutation compared to the MYDSS wild-type [27]. However, the impact of the MYD88 L265P mutation on OS remains controversial, because no association of overall survival with the MYDS8 L265P mutation was reported in another study [28]. In our study, not all patients were evaluated for the MYD88 L265P mutation; thus, we could not analyze the association of early death with the mutation. However, the one analyzed case of early death had the MYD88 L265P mutation as well as mutations in TP53 and ARID1A. The TP53 mutation has been observed in $7.3 \%$ of WM patients who had shorter survival in a previous study [29]. Truncated mutations of ARIDiA have also been reported in WM patients, including single-nucleotide variants leading to premature protein truncation [30]. Although the precise mechanisms by which these mutations influence the occurrence of early death remain to be elucidated, evaluation of mutation profiles at diagnosis might provide helpful information for predicting early death in WM patients, given their crucial role in the pathogenesis of WM (Fig. 4B).

In summary, we analyzed our experience of managing WM patients and evaluated the prognostic relevance of various risk models for WM. Although we analyzed a relatively small number of patients who were heterogeneously treated due to the retrospective nature of the study, our results suggest that serum $\beta 2$-microglobulin level and the IPSS-WM risk model can predict poor survival in WM patients. In addition, mutation analysis might provide additional information on risk models based on clinical and laboratory parameters.

\section{KEY MESSAGE}

1. Serum $\beta 2$-microglobulin level could be a single biomarker strongly predictive of poor survival of Waldenström macroglobulinemia (WM) patients.
2. The low-risk group of the International Prognostic Staging System for WM risk model has better prognostic value than other risk models, and mutation analysis might provide additional information to predict a high-risk group.

\section{Conflict of interest}

No potential conflict of interest relevant to this article was reported.

\section{Acknowledgments}

We would like to thank the patients, their families, and their caregivers who made this study possible. We also thank all the study investigators and study staff.

\section{REFERENCES}

1. Brandefors L, Kimby E, Lundqvist K, Melin B, Lindh J. Familial Waldenstrom's macroglobulinemia and relation to immune defects, autoimmune diseases, and haematological malignancies: a population-based study from northern Sweden. Acta Oncol 2016;55:91-98.

2. Owen RG. Developing diagnostic criteria in Waldenstrom's macroglobulinemia. Semin Oncol 2003;30:196200.

3. Bufalino SB, Patel MM, Kruczek KR, et al. Disease characteristics, treatment patterns, and patient outcomes of lymphoplasmacytic lymphoma or Waldenstroms macroglobulinemia: a single institution retrospective review. Blood 2016;128:5341.

4. Kyle RA, Treon SP, Alexanian R, et al. Prognostic markers and criteria to initiate therapy in Waldenstrom's macroglobulinemia: consensus panel recommendations from the Second International Workshop on Waldenstrom's Macroglobulinemia. Semin Oncol 2003;30:116-120.

5. Zheng YH, Xu L, Cao C, et al. Rituximab-based combination therapy in patients with Waldenstrom macroglobulinemia: a systematic review and meta-analysis. Onco Targets Ther 2019;12:2751-2766.

6. Dimopoulos MA, Tedeschi A, Trotman J, et al. Phase 3 trial of ibrutinib plus rituximab in Waldenström's macroglobulinemia. N Engl J Med 2018;378:2399-2410.

7. Brandefors L, Melin B, Lindh J, Lundqvist K, Kimby E. Prognostic factors and primary treatment for Walden- 
ström macroglobulinemia: a Swedish Lymphoma Registry study. Br J Haematol 2018;183:564-577.

8. Morel P, Duhamel A, Gobbi P, et al. International prognostic scoring system for Waldenstrom macroglobulinemia. Blood 2009;113:4163-4170.

9. Dhodapkar MV, Hoering A, Gertz MA, et al. Long-term survival in Waldenstrom macroglobulinemia: 10-year follow-up of Southwest Oncology Group-directed intergroup trial S9003. Blood 2009;113:793-796.

10. Ghobrial IM, Fonseca R, Gertz MA, et al. Prognostic model for disease-specific and overall mortality in newly diagnosed symptomatic patients with Waldenstrom macroglobulinaemia. Br J Haematol 2006;133:158-164.

11. Morel P, Monconduit M, Jacomy D, et al. Patients with the description of a new scoring system and its validation on 253 other patients. Blood 2000;96:852-858.

12. Bustoros M, Sklavenitis-Pistofidis R, Kapoor P, et al. Progression risk stratification of asymptomatic Waldenström macroglobulinemia. J Clin Oncol 2019;37:1403-1411.

13. Treon SP, Gertz MA, Dimopoulos M, et al. Update on treatment recommendations from the Third International Workshop on Waldenstrom's macroglobulinemia. Blood 2006;107:3442-3446.

14. Hyeon J, Lee B, Shin SH, et al. Targeted deep sequencing of gastric marginal zone lymphoma identified alterations of TRAF3 and TNFAIP 3 that were mutually exclusive for MALT1 rearrangement. Mod Pathol 2018;31:1418-1428.

15. Shin HT, Choi YL, Yun JW, et al. Prevalence and detection of low-allele-fraction variants in clinical cancer samples. Nat Commun 2017;8:1377.

16. Wilm A, Aw PP, Bertrand D, et al. LoFreq: a sequence-quality aware, ultra-sensitive variant caller for uncovering cell-population heterogeneity from high-throughput sequencing datasets. Nucleic Acids Res 2012;40:11189-11201.

17. Cibulskis K, Lawrence MS, Carter SL, et al. Sensitive detection of somatic point mutations in impure and heterogeneous cancer samples. Nat Biotechnol 2013;31:213219.

18. Buske C, Hoster E, Dreyling M, et al. The addition of rituximab to front-line therapy with CHOP (R-CHOP) results in a higher response rate and longer time to treatment failure in patients with lymphoplasmacytic lymphoma: results of a randomized trial of the German Low-Grade Lymphoma Study Group (GLSG). Leukemia 2009;23:153-161.

19. Rummel MJ, Niederle N, Maschmeyer G, et al. Bendamustine plus rituximab versus $\mathrm{CHOP}$ plus rituximab as firstline treatment for patients with indolent and mantle-cell lymphomas: an open-label, multicentre, randomised, phase 3 non-inferiority trial. Lancet 2013;381:1203-1210.

20. Shin SY, Lee ST, Kim HY, et al. Detection of MYD88 L265P in patients with lymphoplasmacytic lymphoma/ Waldenstrom macroglobulinemia and other B-cell non-Hodgkin lymphomas. Blood Res 2016;51:181-186.

21. Kim JM, Ko YH, Lee SS, et al. WHO classification of malignant lymphomas in Korea: report of the third nationwide study. J Pathol Transl Med 2011;45:254-26o.

22. Dimopoulos MA, Kastritis E, Owen RG, et al. Treatment recommendations for patients with Waldenström macroglobulinemia (WM) and related disorders: IWWM-7 consensus. Blood 2014;124:1404-1411.

23. Treon SP. How I treat Waldenström macroglobulinemia. Blood 2015;126:721-732.

24. Milani P, Merlini G. Monoclonal IgM-related AL amyloidosis. Best Pract Res Clin Haematol 2016;29:241-248.

25. Kyle RA, Gertz MA. Primary systemic amyloidosis: clinical and laboratory features in 474 cases. Semin Hematol 1995;32:45-59.

26. Treon SP, Xu L, Yang G, et al. MYD88 L265P somatic mutation in Waldenström's macroglobulinemia. N Engl J Med 2012;367:826-833.

27. Treon SP, Gustine J, Xu L, et al. MYD88 wild-type Waldenstrom macroglobulinaemia: differential diagnosis, risk of histological transformation, and overall survival. Br J Haematol 2018;180:374-380.

28. Abeykoon JP, Paludo J, King RL, et al. MYD88 mutation status does not impact overall survival in Waldenström macroglobulinemia. Am J Hematol 2018;93:187-194.

29. Poulain S, Roumier C, Bertrand E, et al. TP53 mutation and its prognostic significance in Waldenstrom's macroglobulinemia. Clin Cancer Res 2017;23:6325-6335.

30. Hunter ZR, Yang G, Xu L, Liu X, Castillo JJ, Treon SP. Genomics, signaling, and treatment of Waldenström macroglobulinemia. J Clin Oncol 2017;35:994-1001. 
Supplementary Table 1. Gene list included in HemaSCAN

\begin{tabular}{|c|c|c|c|c|c|c|}
\hline$A B L_{1}$ & ACTB & ADGRA2 & $A K T_{1}$ & $A K T_{2}$ & $A K T_{3}$ & $A L K$ \\
\hline$A M E R_{1}$ & ANKRD11 & $A P C$ & APHıA & $A R$ & $A R A F$ & $A R F R P_{1}$ \\
\hline $\mathrm{ARHGAP}_{2} 6$ & ARIDıA & ARID2 & ASXL1 & ATM & ATR & ATRX \\
\hline AURKA & $A U R K B$ & AXIN1 & $A X L$ & $\mathrm{~B}_{2} \mathrm{M}$ & $B A P_{1}$ & $B A R D 1$ \\
\hline BCL10 & $B C L_{11} B$ & BCL2 & $B C L 2 L_{2}$ & BCL6 & $B C L j A$ & BCOR \\
\hline BCORL1 & $B C R$ & $\mathrm{BIRC}_{3}$ & BLM & BRAF & $B R C A 1$ & BRCA2 \\
\hline $\mathrm{BRD}_{4}$ & $B R I P_{1}$ & BRSK1 & BTG1 & BTG2 & BTK & BTLA \\
\hline$C A D$ & CARD11 & CBFB & CBL & CCND1 & $\mathrm{CCND}_{2}$ & $\mathrm{CCND}_{3}$ \\
\hline CCNE1 & ССТ6В & $C D 22$ & $\mathrm{CD} 274$ & $\mathrm{CD} 2 \mathrm{~S}$ & $\mathrm{CD}_{3} 6$ & $\mathrm{CD}_{5} 8$ \\
\hline$C D_{\text {jo }}$ & $C D_{79} A$ & $\mathrm{CD}_{79} \mathrm{~B}$ & $\mathrm{CDC}_{73}$ & $\mathrm{CDH} 1$ & CDK12 & $\mathrm{CDK}_{4}$ \\
\hline CDK6 & CDKS & $C D K N ı B$ & CDKN2A & $\mathrm{CDKN} 2 \mathrm{~B}$ & $\mathrm{CDKN}_{2} \mathrm{C}$ & CEBPA \\
\hline $\mathrm{CHD}_{2}$ & CHEK 1 & CHEK2 & CIC & CIITA & $C K S_{1} B$ & $\mathrm{CPS}_{1}$ \\
\hline CREBBP & CRKL & CRLF2 & $\mathrm{CSF}_{1} \mathrm{R}$ & $\mathrm{CSF}_{3} \mathrm{R}$ & CTCF & CTNNA1 \\
\hline CTNNB $_{1}$ & $C U X_{1}$ & $\mathrm{CXCR}_{4}$ & $D A X X$ & $\mathrm{DDR} 2$ & $\mathrm{DDX}_{3} \mathrm{X}$ & DNM2 \\
\hline $\mathrm{DNMT}_{3} \mathrm{~A}$ & $\mathrm{DOT}_{1} L$ & $D T X_{1}$ & DUSP2 & DUSPg & $E B F_{1}$ & $E C T_{2} L$ \\
\hline EED & EGFR & $E L P 2$ & EMSY & $E P_{300}$ & ЕРНА3 & EPHA5 \\
\hline EPHAJ & $E P H B 1$ & EPOR & $E R B B 2$ & $E R B B_{3}$ & $E R B B_{4}$ & $E R G$ \\
\hline ESR1 & $E S_{1}$ & ETV1 & $E_{T V}$ & ETV $_{5}$ & ETV6 & $E W S R 1$ \\
\hline EXOSC6 & $\mathrm{EZH} 2$ & FAF1 & $\mathrm{FAM}_{4} 6 \mathrm{C}$ & FANCA & FANCC & FANCD2 \\
\hline FANCE & FANCF & FANCG & FANCL & FAS & FBXO11 & $\mathrm{FBXO}_{31}$ \\
\hline $\mathrm{FBXW}_{7}$ & FGF10 & FGF14 & FGF19 & $F_{F F} 23$ & $F_{F F}$ & $\mathrm{FGF}_{4}$ \\
\hline FGF6 & FGFR 1 & FGFR2 & $\mathrm{FGFR}_{3}$ & FGFR4 & FHIT & FLCN \\
\hline FLT1 & $\mathrm{FLT}_{3}$ & $\mathrm{FLT}_{4}$ & FLYWCH1 & FOXL2 & FOXO1 & $\mathrm{FOXO}_{3}$ \\
\hline FOXP1 & $F R S 2$ & FYN & $\mathrm{GADD}_{45} \mathrm{~B}$ & GATA1 & GATA2 & $\mathrm{GATA}_{3}$ \\
\hline $\mathrm{GID}_{4}$ & GNA11 & GNA12 & GNA13 & GNAQ & GNAS & GRIN2A \\
\hline $\mathrm{GSK}_{3} \mathrm{~B}$ & GTSE$_{1}$ & $H D A C 1$ & $\mathrm{HDAC}_{4}$ & $\mathrm{HDAC}_{7}$ & HGF & $\mathrm{HIST}_{1} \mathrm{H}_{1} \mathrm{C}$ \\
\hline $\mathrm{HIST}_{1} \mathrm{H}_{1} \mathrm{D}$ & $\mathrm{HIST}_{1} \mathrm{H}_{1} \mathrm{E}$ & $\mathrm{HIST}_{1} \mathrm{H}_{2} \mathrm{AC}$ & $\mathrm{HIST}_{1} \mathrm{H}_{2} \mathrm{AG}$ & $\mathrm{HIST}_{1} \mathrm{H}_{2} \mathrm{AL}$ & $\mathrm{HIST}_{1} \mathrm{H}_{2} \mathrm{AM}$ & $\mathrm{HIST}_{1} \mathrm{H}_{2} \mathrm{BC}$ \\
\hline $\mathrm{HIST}_{1} \mathrm{H}_{2} \mathrm{BJ}$ & $\mathrm{HIST}_{1} \mathrm{H}_{2} \mathrm{BK}$ & $\mathrm{HIST}_{1} \mathrm{H}_{2} \mathrm{BO}$ & $\mathrm{HIST}_{1} \mathrm{H}_{3} \mathrm{~B}$ & HNF1A & HRAS & $\mathrm{HSP}_{9 \circ A A 1}$ \\
\hline ICK & $I D_{3}$ & $I D H_{1}$ & $\mathrm{IDH}_{2}$ & $I_{G F} R$ & IKBKE & $I K Z F 1$ \\
\hline IKZF2 & $I_{K Z F}$ & IL2oRA & $I L J R$ & INHBA & $\mathrm{INPP}_{4} \mathrm{~B}$ & $I N P_{5} D$ \\
\hline$I R F 1$ & $I_{R} F_{4}$ & IRFS & IRS 2 & JAK1 & JAK2 & $\mathrm{JAK}_{3}$ \\
\hline JARID2 & JUN & KAT6A & $\mathrm{KDM}_{2} \mathrm{~B}$ & $\mathrm{KDM}_{4} \mathrm{C}$ & $\mathrm{KDM}_{5} \mathrm{~A}$ & $\mathrm{KDM}_{5} \mathrm{C}$ \\
\hline KDM6A & $K D R$ & $K E A P_{1}$ & KIT & KLHLG & KRAS & $L E F 1$ \\
\hline LILRB1 & $L R P_{1} B$ & LRRK2 & MAF & MAFB & MAGED1 & MALT1 \\
\hline $\mathrm{MAP}_{2} \mathrm{~K} 1$ & $M A P_{2} K_{2}$ & $\mathrm{MAP}_{2} \mathrm{~K}_{4}$ & $\mathrm{MAP}_{3} \mathrm{~K}_{1}$ & $\mathrm{MAP}_{3} \mathrm{~K} 14$ & $\mathrm{MAP}_{3} \mathrm{~K} 6$ & $\mathrm{MAP}_{3} \mathrm{~K}_{7}$ \\
\hline MAPK1 & MCL1 & MDM2 & $\mathrm{MDM}_{4}$ & $M E D 12$ & $\mathrm{MEF} 2 \mathrm{~B}$ & $\mathrm{MEF} 2 \mathrm{C}$ \\
\hline MENı & MET & MIB1 & MITF & MKI67 & MLH1 & MPL \\
\hline MRE11A & $\mathrm{MSH}_{2}$ & $\mathrm{MSH}_{3}$ & MSH6 & MTOR & $M U C_{2}$ & MUTYH \\
\hline
\end{tabular}


KJIM'

Supplementary Table 1. Continued

\begin{tabular}{|c|c|c|c|c|c|c|}
\hline MrC & MYCL & MYCN & MYDSS & MYOıSA & NCOR2 & NCSTN \\
\hline$N F_{1}$ & $\mathrm{NF}_{2}$ & NFE2L2 & NFKBIA & $N K X_{2-1}$ & $N_{1}$ & $\mathrm{NOTCH}_{1}$ \\
\hline $\mathrm{NOTCH}_{2}$ & NPMı & NRAS & $\mathrm{NT}_{5} \mathrm{C}_{2}$ & $N T R K 1$ & NTRK2 & NTRK3 \\
\hline $\mathrm{NUP}_{93}$ & NUPgS & P2RYS & PAG1 & $\mathrm{PAK}_{3}$ & PALB2 & PASK \\
\hline $\mathrm{PAX}_{5}$ & PBRM1 & $P C$ & PCBP1 & PCLO & PDCD 1 & PDCD11 \\
\hline PDCD1LG2 & PDGFRA & PDGFRB & PDK1 & PHF6 & $\mathrm{PIK}_{3} \mathrm{CA}$ & $\mathrm{PIK}_{3} \mathrm{CG}$ \\
\hline $\mathrm{PIK}_{3} \mathrm{R}_{1}$ & $\mathrm{PIK}_{3} \mathrm{R}_{2}$ & PIM1 & PLCG1 & PLCG2 & POT1 & $\mathrm{POU}_{2} \mathrm{~F}_{2}$ \\
\hline$P P P_{2} R_{1} A$ & PRDM1 & PRKARıA & PRKDC & PRSSS & PTCHı & PTEN \\
\hline PTPN11 & PTPN2 & PTPNG & PTPRO & $R A D_{21}$ & $R A D_{50}$ & $R A D_{51}$ \\
\hline RAF1 & RARA & RASGEF1A & $R B 1$ & RELN & RET & RHOA \\
\hline $\mathrm{RHOT}_{2}$ & RICTOR & $\mathrm{RNF}_{43}$ & $\mathrm{ROS}_{1}$ & RPTOR & $R_{U N X_{1}}$ & $\mathrm{~S}_{1} \mathrm{PR} 2$ \\
\hline SDHA & SDHB & SDHC & SDHD & $\mathrm{SERP}_{2}$ & SETBP1 & SETD2 \\
\hline $\mathrm{SF}_{3} \mathrm{~B}_{1}$ & $S G K 1$ & SMAD2 & $\mathrm{SMAD}_{4}$ & SMARCA1 & $\mathrm{SMARCA}_{4}$ & SMARCAL1 \\
\hline$S M A R C B 1$ & SMARCD1 & $S M C_{1} A$ & $S M C_{3}$ & SMO & $S_{O C S}$ & $\mathrm{SOCS}_{2}$ \\
\hline $\mathrm{SOCS}_{3}$ & SOX10 & $\mathrm{SOX}_{2}$ & SPEN & SPOP & $S R C$ & SRSF2 \\
\hline STAG2 & STAT1 & STAT2 & $\mathrm{STAT}_{3}$ & $\mathrm{STAT}_{4}$ & $\operatorname{STAT}_{5} A$ & $\operatorname{STAT}_{5} B$ \\
\hline STAT6 & STK11 & SUFU & $S U Z_{12}$ & $T_{A F}$ & $T_{B L} X_{1} R_{1}$ & $\mathrm{TCF}_{3}$ \\
\hline TCL1A & TET2 & $\mathrm{TET}_{3}$ & TGFBR2 & TLL2 & TMEM3०A & TMPRSS2 \\
\hline TNFAIP $_{3}$ & TNFRSF11A & TNFRSF14 & TNFRSF 17 & $\mathrm{TOP}_{1}$ & $T P_{53}$ & TP63 \\
\hline TRAF2 & $\mathrm{TRAF}_{3}$ & $T_{R A F_{5}}$ & $T_{S C}$ & $\mathrm{TSC}_{2}$ & TSHR & TUSC $_{3}$ \\
\hline TYK2 & $U_{2} A F_{1}$ & $U_{2} A F 2$ & $V A V_{1}$ & VHL & WDRgo & $\mathrm{WHSC}_{1}$ \\
\hline WIF1 & WISP $_{3}$ & $W T_{1}$ & WWOX & $X B P_{1}$ & XPO1 & $\Upsilon_{1} A P_{1}$ \\
\hline ZMYM3 & ZNF217 & ZNF24 & ZNF703 & ZRSR2 & & \\
\hline
\end{tabular}

